\title{
Modelos não lineares para a liberação de potássio de estercos animais em latossolos
}

\author{
Non linear models to potassium release from animals manure in Latosols
}

\author{
Walmes Marques Zeviani ${ }^{\mathrm{I}}$ Carlos Alberto Silva ${ }^{\mathrm{II}}$ Waldete Japiassu de Oliveira Carneiro ${ }^{\mathrm{II}}$ \\ Joel Augusto Muniz ${ }^{\text {V }}$
}

RESUMO

Modelos não lineares são adequados para a descrição da liberação de nutrientes, uma vez que estimam quantidades de interesse prático e apresentam boa qualidade de ajuste. Embora seu processo inferencial seja baseado em argumentos assintóticos, existem meios de se conhecer a intensidade da não linearidade. Neste trabalho, avaliou-se a não linearidade de dois modelos de regressão não linear por meio das curvaturas de Bates e Watts, vício de Box e do estudo das propriedades amostrais dos estimadores de mínimos quadrados, obtido por simulação. Os dados são provenientes do estudo, ao longo do tempo, da liberação de $K$ de quatro estercos animais em combinação com dois solos. $O$ modelo Exponencial foi mais adequado, em termos inferenciais e para aplicação prática, uma vez que por todas as medidas apresentou menor não linearidade.

Palavras-chave: curvatura de Bates e Watts, vício de Box, bootstrap, meia vida.

\section{ABSTRACT}

Nonlinear models are appropriate to describe nutrient release, since they estimate quantities of practical interest and they have goodness of fit. Although its inferential process is based on asymptotic arguments, there are ways to know the nonlinearity intensity. In this work, we evaluate the nonlinearity of two nonlinear regression models through the curvatures of Bates e Watts, bias of Box and the least squares estimator sampling properties by simulation study. The data are from the study, over time, of the $K$ release from 4 animal manure in combination with 2 soils. The exponential model was more appropriate in terms of inferential and practical aspects, since by all measures showed lower nonlinearity.

Key words: curvature of Bates e Watts, bias of Box, bootstrap, half-life.

\section{INTRODUÇÃO}

Entre os macronutrientes, o potássio (K) é o segundo exigido em maior quantidade pelas plantas superiores (MALAVOLTA, 1980). Entretanto, tais níveis de exigência contrastam, em geral, com os teores insuficientes desse elemento em alguns solos brasileiros (NACHTIGALL \& RAIJ, 2005). Devido ao fato do $\mathrm{K}$ desempenhar inúmeras funções na planta, o fornecimento desse nutriente deve ser feito em quantidades corretas, o que pode ser conseguido tanto pelo emprego de fontes minerais quanto orgânicas.

Vários estudos demonstram que a adubação orgânica tem uma atuação muito positiva sobre as mudas de diferentes espécies frutíferas. Tal fato tem estimulado muitos produtores a adotarem essa prática, que tem como vantagem o uso de material orgânico, muitas vezes, disponível na propriedade, além de agregar valor ao produto (CANESIN \& CORREA, 2006).

A aplicação de modelos que proporcionem elevado grau de explicação, sobre o fenômeno de

'Programa de Pós-graduação em Estatística e Experimentação Agropecuária, Universidade Federal de Lavras (UFLA), 37200-

000, Lavras, MG, Brasil. E-mail: walmes@ufpr.br. Autor para correspondência.

IIDepartamento de Ciência do Solo, UFLA, Lavras, MG, Brasil.

IIIPrograma de Pós-graduação em Ciência do Solo, UFLA, Lavras, MG, Brasil.

${ }^{\text {IV }}$ Departamento de Ciências Exatas, UFLA, Lavras, MG, Brasil. 
liberação de $K$, assegura maior precisão na recomendação de $\mathrm{K}$ orgânico em complemento ao $\mathrm{K}$ fornecido por fontes minerais. Ao contrário do N, descrito por PEREIRA et al. (2005), o padrão de liberação do $\mathrm{K}$ de fontes orgânicas ainda é pouco conhecido e isso se configura numa forte limitação ao aproveitamento de fontes orgânicas para o fornecimento desse nutriente.

Processos como de liberação de nutrientes podem ser bem caracterizados por modelos de regressão (CAMARGO et al., 2002; PEREIRA et al., 2005). Em geral, tal processo ocorre de forma que a liberação de todo o nutriente disponível ocorra a taxas decrescentes, à medida que o nutriente é liberado, o que caracteriza um comportamento não linear.

Muitos dos modelos apresentados na literatura são não lineares e, portanto, cuidados devem ser tomados ao se fazer inferências (e.g. construção de intervalos de confiança e testes de hipóteses) (MAZUCHELI \& ACHCAR, 2002; BARROZO et al., 2004, MENDES et al., 2008). O processo inferencial em modelos não lineares é baseado em argumentos assintóticos (grandes amostras) e é fortemente influenciado pela não linearidade do modelo. Para uma avaliação da qualidade das inferências, vários pesquisadores propuseram medidas para quantificar a não linearidade e avaliar as suas consequências.

Para estimar o vício dos estimadores de mínimos quadrados dos parâmetros de um modelo não linear univariado, BOX (1971) propôs uma fórmula dada por: $V(\hat{\theta})=-\frac{\sigma^{2}}{2}\left(F^{\prime} F\right)^{-1} F^{\prime} d$, em que $V(\hat{\theta})$ representa a diferença entre o vetor de estimativas e dos valores paramétricos, $F$ e $H$ são a matriz jacobiana e o arranjo hessiano das derivadas do modelo $f\left(\theta, x_{i}\right)$ com relação aos parâmetros $(\hat{\theta})$, da seguinte forma:

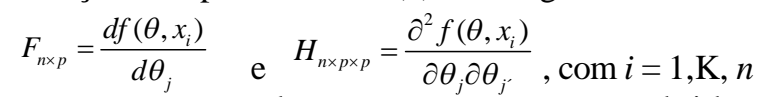
e $j=1, \mathrm{~K}, p$, sendo $d$ um vetor de traços obtidos por $d_{i}=\operatorname{tr}\left[\left(F^{\prime} F\right)^{-1} H_{i . .}\right]$. Na prática, são usadas as estimativas $\hat{\sigma}^{2}$ e $\hat{\theta}$ no lugar dos parâmetros desconhecidos.

Na avaliação de modelos não lineares, o autor sugere apresentar as estimativas dos vícios em termos percentuais da estimativa, ou seja, $\% V(\hat{\theta})=100 \cdot V(\hat{\theta}) / \hat{\theta}$, e afirma que valores de $\% V(\hat{\theta})$ acima de $1 \%$, em valor absoluto, indicam comportamento não linear. A importância de se avaliar o vício reside em indicar quais parâmetros do modelo mais contribuem para o afastamento do comportamento linear. O emprego de simulação computacional foi proposta por RATKOWSKY (1983) para estudar a distribuição amostral de estimadores de parâmetros a fim de quantificar a não linearidade bem como sugerir reparametrizações para o modelo. $\mathrm{O}$ autor menciona a aplicação de testes formais de assimetria, curtose e normalidade para as amostras obtidas da simulação para cada parâmetro, além da avaliação de histogramas de frequência, de forma que parâmetros que apresentarem distribuição amostral próxima da normal indicam comportamento linear do modelo.

Medidas de não linearidade, baseadas no conceito geométrico de curvatura, foram apresentadas por BATES \& WATTS (1980). Essas medidas independem da escala da variável resposta e são úteis tanto para avaliar o comportamento de diferentes conjuntos de dados como de reparametrizações de um mesmo modelo. Os autores decompuseram a não linearidade em duas componentes: a não linearidade intrínseca (IN) e a não linearidade devido ao efeito de parâmetros (EP). A IN mede a falta de planicidade da superfície esperada, gerada pelo modelo na vizinhança do local da solução de mínimos quadrados e não se altera devido à reparametrizações do modelo. A EP mede a falta de uniformidade do sistema de coordenadas da superfície esperada na vizinhança do local da solução e é dependente da parametrização do modelo. De acordo com SEBER \& WILD (1989), quanto menor o valor das duas componentes da não linearidade mais próximo, o modelo não linear estará do linear em que essas quantidades são zero. Entre as características de uma boa aproximação linear, está a garantia de estimadores não viesados, normalmente distribuídos, com variância mínima mesmo em pequenas amostras (SEBER \& WILD, 1989; MAZUCHELI \& ACHCAR, 2002).

O objetivo do estudo foi avaliar os modelos de liberação de K de resíduos orgânicos de origem animal quanto a não linearidade por meio das técnicas: vício de Box, curvatura de Bates e Watts e pelas propriedades amostrais dos estimadores via estudo de simulação.

\section{MATERIAL E MÉTODOS}

Os dados foram obtidos de OLIVEIRA (2008) e se referem ao conteúdo de $\mathrm{K}$ liberado, em função do tempo, por quatro resíduos orgânicos de origem animal, incubados em dois solos. Os resíduos estudados foram esterco bovino (EB), esterco de codorna (EC), esterco equino (EE) e esterco de galinha (EG), que foram incubados em Latossolo Vermelho Amarelo (LVA) e Latossolo Vermelho distroférrico (LVdf), na quantidade de 207, 284, 153 e 166 $\mathrm{mg} \mathrm{kg}^{-1}$ de solo, respectivamente.

$\mathrm{O}$ estudo da dinâmica de liberação do $\mathrm{K}$ foi realizado por meio da coleta periódica de lixiviados, a cada 15 dias, durante os três primeiros meses de incubação e a 
cada 30 dias, até o final do experimento, perfazendo 12 coletas (270 dias). O delineamento experimental foi inteiramente causalizado com três repetições para cada combinação de solo-esterco, perfazendo portanto 288 coletas de K durante o período experimental.

Avaliou-se o modelo Michaelis-Menten acrescido de uma constante $K=A . t /(V+t)+D . t$ (Quociente) e o modelo de CABRERA (1993) reparametrizado $K=A .(1-\exp (-\ln (2) \cdot t / V)+D \cdot t$ (Exponencial). Para este trabalho, os modelos foram obtidos pela adequação dos modelos originais para que se estimassem diretamente os parâmetros: conteúdo total de K prontamente liberável ( $A$, assíntota, $\mathrm{mg})$, tempo necessário para a liberação de metade $(A / 2$, meia vida) do $\mathrm{K}$ da porção prontamente liberável ( $V$, dias) e taxa de liberação do $\mathrm{K}$ da porção de lenta liberação $\left(D, \mathrm{mg} \mathrm{kg}^{-1} \mathrm{dia}^{-1}\right)$. Dessa forma, considerando os modelos apresentados, a liberação do potássio prontamente liberável é descrita pelos parâmetros $A \mathrm{e}$ $V$ e o potássio de lenta liberação pelo parâmetro $D$.

A avaliação da não linearidade baseou-se na quantificação do vício relativo de Box (BOX, 1971), das curvaturas médias (BATES \& WATTS, 1980) e no estudo de simulação (RATKOWSKY, 1983). A significância estatística dos valores de curvatura $(\gamma)$ foi comparada com oraio da região de confiança, $100(1-\alpha)$ em que $\gamma \sqrt{F_{(\alpha, p, n-p)}}<0,5$ é um indicativo de nãolinearidade para um nível $a$ de significância, considerando uma distribuição F com p e n-p graus de liberdade (MAZUCHELI \&ACHCAR, 2002). No estudo de simulação bootstrap, foram simulados 3000 experimentos em que se tomaram as estimativas dos parâmetros $\bar{\theta}$ e do erro aleatório $\hat{\sigma}$ como os valores paramétricos reais. Nas amostras bootstrap obtidas, foram aplicados teste de assimetria e curtose para cada parâmetro. $\mathrm{O}$ vício relativo bootstrap foi calculado por
$\% V_{b}(\hat{\theta})=100(\bar{\theta}-\hat{\theta}) / \hat{\theta}$, em que $\bar{\theta}$ é a média das 3000 estımatıvas obtıdas na simulação.

A qualidade do ajuste foi avaliada pelo teste da falta de ajuste e pelo coeficiente de determinação $\left(\mathrm{R}^{2}\right)$. A qualidade dos estimadores foi avaliada pelo comprimento do intervalo de confiança obtido para as estimativas dos parâmetros. Considerou-se um nível nominal de significância (a) de 5\% para todas as inferências aplicadas. Todo desenvolvimento computacional foi feito com o aplicativo R 2.9.0 (R DEVELOPMENT CORE TEAM, 2009). Detalhes em ZEVIANI (2009).

\section{RESULTADOS E DISCUSSÃO}

Os coeficientes de determinação ajustados $\left(\mathrm{R}^{2}\right)$ do ajuste dos modelos aos dados experimentais foram superiores a 95\% (Tabela 1). O teste da falta de ajuste apontou que os modelos são adequados para a descrição da liberação de K para todos os tratamentos, com exceção para o modelo Exponencial ajustado ao LVdfEB, em que se observou um valor $F$ igual 2,50, sendo o $F$ crítico do teste igual a 2,30. Tanto pelo RQMR quanto pelo $\mathrm{R}^{2}$, teve-se que o modelo Quociente apresentou melhor ajuste, na média dos oito tratamentos, embora essa diferença possa ser interpretada como muito pequena sob consideração prática.

A curvatura intrínseca (IN) para ambos os modelos não excedeu a cota de 0,5 , para nenhum tratamento, o que indica adequada aproximação da superfície esperada por um plano na vizinhança da solução de mínimos quadrados (Tabela 1). Embora não excedessem o valor 0,5 , os valores de IN, para o modelo Exponencial, foram superiores, se comparados ao modelo Quociente. O modelo Exponencial apresentou curvatura devido ao efeito de parâmetros (EP) inferior

Tabela 1 - Valores da raiz do quadrado médio do resíduo (RQMR), coeficiente de determinação ajustado ( $\left.\mathrm{R}^{2}\right)$ e da curvatura intrínseca (IN) e devido ao efeito de parâmetros (EP) para cada tratamento, segundo os modelos considerados.

\begin{tabular}{|c|c|c|c|c|c|c|c|c|}
\hline \multirow{2}{*}{ Tratamento } & \multicolumn{2}{|c|}{ 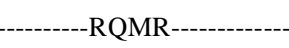 } & $R^{2}$ & & \multirow[b]{2}{*}{ Quo } & \multirow[b]{2}{*}{$\operatorname{Exp}$} & \multirow[b]{2}{*}{ Quo } & \multirow[b]{2}{*}{ Exp } \\
\hline & Quo & Exp & Quo & Exp & & & & \\
\hline LVAEB & 3,728 & 3,737 & 0,998 & 0,997 & 0,179 & 0,355 & 0,589 & 0,363 \\
\hline LVdfEB & 3,521 & 3,960 & 0,972 & 0,950 & 0,071 & 0,154 & 0,235 & 0,159 \\
\hline LVAEC & 8,826 & 8,871 & 0,999 & 0,995 & 0,116 & 0,255 & 0,384 & 0,233 \\
\hline LVdfEC & 11,186 & 11,168 & 0,993 & 0,994 & 0,153 & 0,215 & 0,651 & 0,360 \\
\hline LVAEE & 6,972 & 6,960 & 0,995 & 0,995 & 0,104 & 0,203 & 0,341 & 0,209 \\
\hline LVdfEE & 7,772 & 7,830 & 0,995 & 0,994 & 0,105 & 0,124 & 0,774 & 0,363 \\
\hline LVAEG & 7,107 & 7,113 & 0,996 & 0,996 & 0,169 & 0,330 & 0,555 & 0,339 \\
\hline LVdfEG & 6,143 & 6,119 & 0,993 & 0,994 & 0,111 & 0,170 & 0,406 & 0,241 \\
\hline
\end{tabular}

$\sqrt{F_{(0,05 ; 3 ; 33)}}=1,0074$ 
a 0,5 para todos os tratamentos, ao passo que o modelo Quociente apresentou valor superior a este para os tratamentos LVAEB, LVdfEC, LVdfEE eLVAEG (Tabela 1). Tais resultados indicam que a aproximação da superfície esperada por um plano com coordenadas igualmente espaçadas é adequada para o modelo Exponencial (SEBER \& WILD, 1989), mas nem sempre adequada para o modelo Quociente. BATES \& WATTS (1980) consideraram que é possível minimizar a EP com o emprego de uma reparametrização, mas argumentaram que a escolha da reparametrização pode não ser única, pois é dependente do conjunto de dados.

$\mathrm{O}$ valor predito de $\mathrm{K}$ liberado $(\hat{K})$ ao longo tempo foi próximo para ambos os modelos (Figura 1). Em termos de precisão, quanto à predição do $\mathrm{K}$ liberado, os modelos são equivalentes, uma vez que a amplitude das bandas de confiança ( $I C(\hat{K}))$, ao longo da curva, foi, em termos práticos, de mesmo tamanho. O K médio observado ao longo do tempo $(\bar{K})$ esteve contido dentro da banda de confiança para o valor predito pelo modelo de regressão. No caso particular do modelo Exponencial ajustado ao LVdfEB, é possível observar, pela figura 1, que a falta de ajuste está associada à presença de alguns pontos fora da banda de confiança.
Os intervalos de confiança para os parâmetros do modelo Exponencial apresentaram menor amplitude, comparados ao modelo Quociente (Figura 2). Tal resultado é decorrente do menor padrão do estimador de parâmetros por mínimos quadrados para o modelo Exponencial (Tabela 2 e 3 ).

Embora o valor pontual estimado para os parâmetros em cada modelo seja diferente, para um mesmo tratamento (Tabela 2 e 3), a sobreposição dos intervalos de confiança para o valor estimado não indicou que tais estimativas são significativamente diferentes entre os modelos (Figura 2).

$\mathrm{O}$ valor estimado e o intervalo de confiança obtido por simulação bootstrap indicaram a presença de vício nas estimativas de mínimos quadrados, uma vez que a diferença entre a estimativa de mínimos quadrados e média das amostras boostratp foi diferente de zero (Figura 2). Observou-se que o intervalo de confiança bootstrap foi translocado na mesma direção do vício, embora, de maneira geral, a amplitude dos intervalos de confiança assintóticos e bootstrap apresentaram aproximadamente o mesmo tamanho, o que indica não haver indicativo de excesso de variância para os estimadores de mínimos quadrados (RATKOWSKY, 1983).

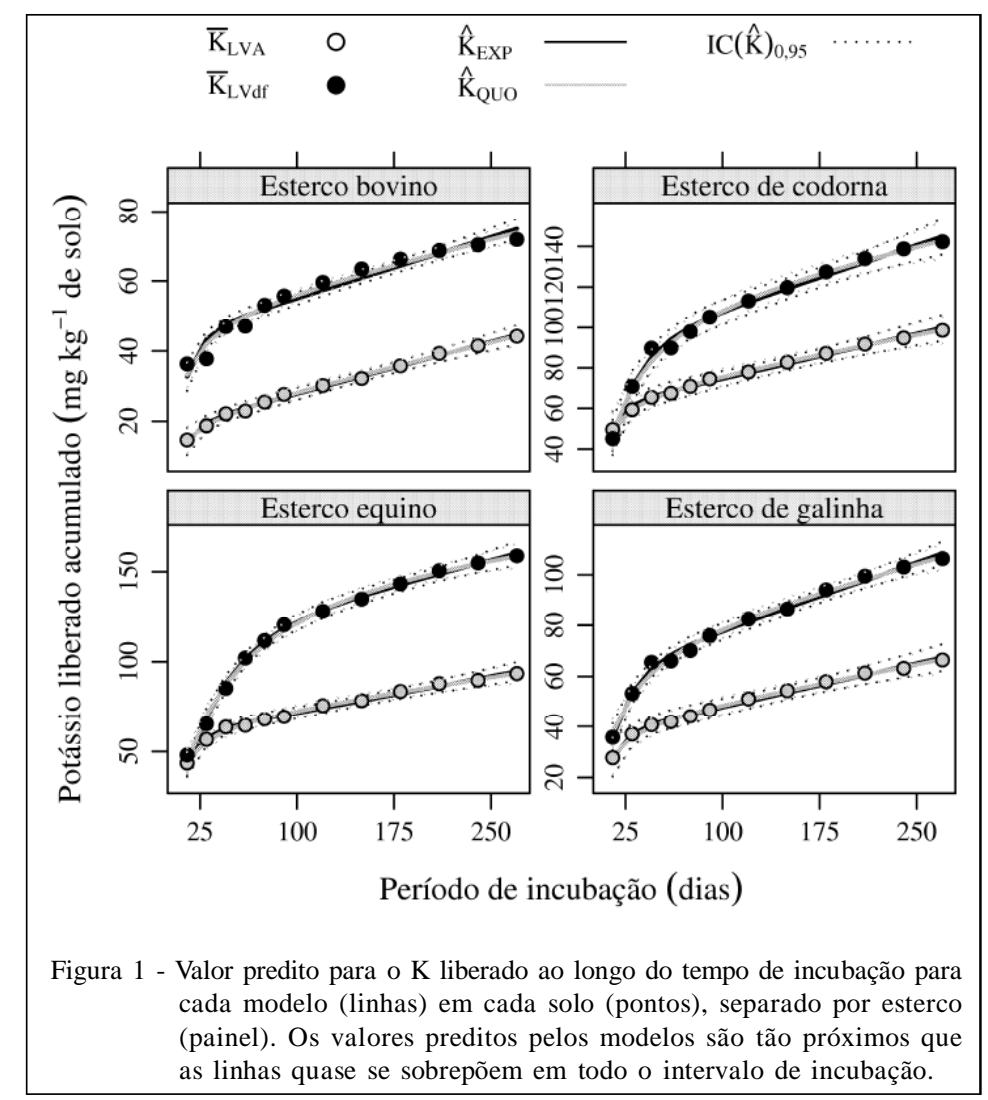

Ciência Rural, v.42, n.10, out, 2012. 


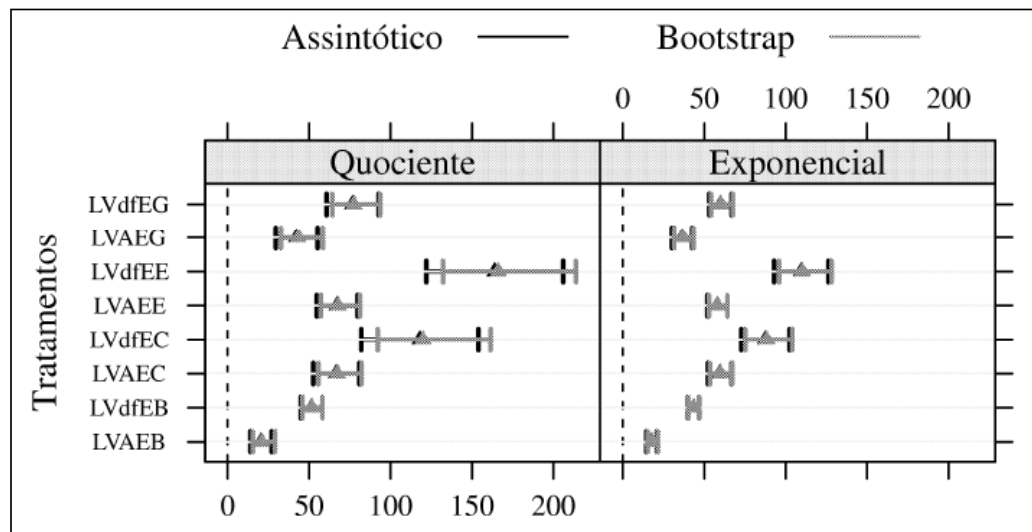

Potássio prontamente liberável $\left(A, \mathrm{mg} \mathrm{kg}^{-1}\right.$ de solo)
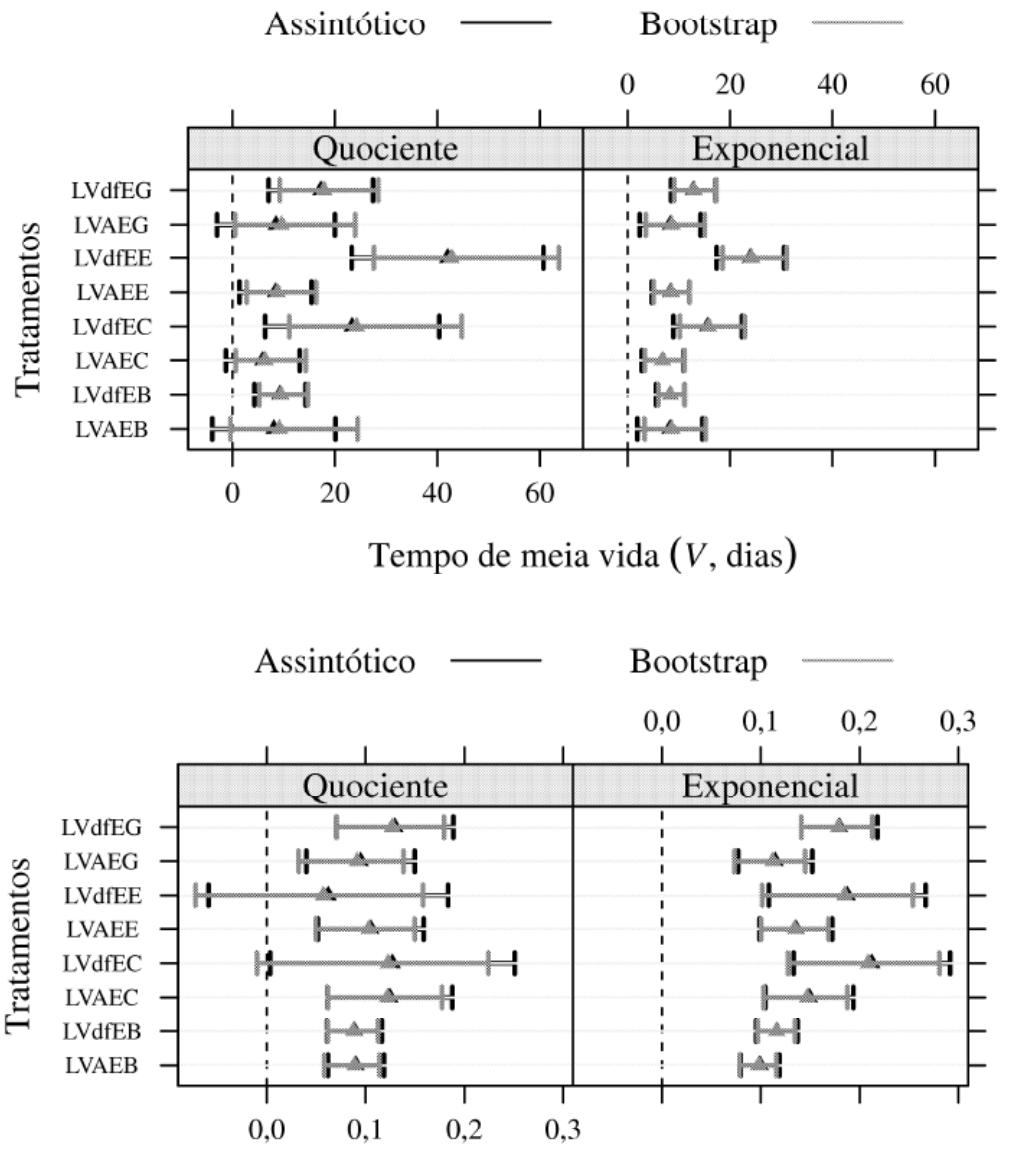

Taxa de lenta liberação $\left(D, \mathrm{mg} \mathrm{kg}^{-1} \mathrm{dia}^{-1}\right)$

Figura 2 - Sobreposição das estimativas intervalares assintóticas e bootstrap para cada parâmetro $(A, V$ e $D)$, em função dos modelos ajustados a cada tratamento.

As estimativas pontuais e intervalares, obtidas pelo método assintótico e bootstrap, foram mais próximas para o modelo Exponencial (Figura 2). GILLS \& RATKOWSKY (1978) apontaram que o grau de aproximação entre os resultados assintóticos e bootstrap pode ser considerado como uma medida de linearidade, uma vez que uma exata sobreposição dos resultados desses métodos é esperada para modelos lineares. 
Tabela 2 - Estimativas, erro padrão, vício de Box, vício bootstrap, assimetria e curtose para os parâmetros em função dos tratamentos para o solo LVA.

\begin{tabular}{|c|c|c|c|c|c|c|}
\hline Mod.-Trat. & Parm. & Est(Err.Pad.) & V.Box $(\%)$ & V.Boot $(\%)$ & Assim. & Curto. \\
\hline \multirow{3}{*}{ Q-EB } & A & $20,496(3,248) * *$ & 2,41 & 2,63 & $0,916 * *$ & $2,089 * *$ \\
\hline & $\mathrm{V}$ & $8,101(5,911) \mathrm{ns}$ & 13,17 & 13,46 & $1,080 * *$ & $2,278 * *$ \\
\hline & $\mathrm{D}$ & $0,090(0,014)^{* *}$ & $-1,62$ & $-1,69$ & $-0,502 * *$ & $0,800 * *$ \\
\hline \multirow{3}{*}{ E-EB } & A & $17,790(1,614)^{* *}$ & 1,13 & 1,22 & $0,446^{* *}$ & $0,356^{* *}$ \\
\hline & $\mathrm{V}$ & $8,251(3,114)^{*}$ & 3,13 & 3,21 & $0,790 * *$ & $1,376^{* *}$ \\
\hline & $\mathrm{D}$ & $0,099(0,010)^{* *}$ & $-0,97$ & $-1,06$ & $-0,175^{*}$ & $-0,004 \mathrm{~ns}$ \\
\hline \multirow{3}{*}{ Q-EC } & A & $66,719(6,934)^{* *}$ & 1,01 & 1,12 & $0,429 * *$ & $0,305^{* *}$ \\
\hline & $\mathrm{V}$ & $5,925(3,538) \mathrm{ns}$ & 7,18 & 7,27 & $0,770 * *$ & $1,056^{* *}$ \\
\hline & $\mathrm{D}$ & $0,125(0,031)^{* *}$ & $-1,66$ & $-1,85$ & $-0,165^{*}$ & $0,029 \mathrm{~ns}$ \\
\hline \multirow{3}{*}{ E-EC } & A & $59,616(3,578) * *$ & 0,47 & 0,71 & $0,131 \mathrm{~ns}$ & $-0,001 \mathrm{~ns}$ \\
\hline & $\mathrm{V}$ & $6,862(2,013)^{* *}$ & 1,15 & 1,84 & $0,351 * *$ & $0,496 * *$ \\
\hline & $\mathrm{D}$ & $0,149(0,022)^{* *}$ & $-0,93$ & $-1,56$ & $-0,069 \mathrm{~ns}$ & $-0,036 \mathrm{~ns}$ \\
\hline \multirow{3}{*}{ Q-EE } & A & $67,173(6,161)^{* *}$ & 0,81 & 1,02 & $0,432 * *$ & $0,249 *$ \\
\hline & $\mathrm{V}$ & $8,410(3,462)^{*}$ & 4,29 & 5,01 & $0,485^{* *}$ & $0,356 * *$ \\
\hline & $\mathrm{D}$ & $0,105(0,026) * *$ & $-1,52$ & $-2,12$ & $-0,211 * *$ & $0,026 \mathrm{~ns}$ \\
\hline \multirow{3}{*}{ E-EE } & A & $58,057(3,029) * *$ & 0,37 & 0,28 & $0,171^{*}$ & $0,005 \mathrm{~ns}$ \\
\hline & $\mathrm{V}$ & $8,395(1,796)^{* *}$ & 1,04 & 0,08 & $0,083 \mathrm{~ns}$ & $-0,029 \mathrm{~ns}$ \\
\hline & $\mathrm{D}$ & $0,136(0,018)^{* *}$ & $-0,77$ & $-0,64$ & $0,019 \mathrm{~ns}$ & $-0,105 \mathrm{~ns}$ \\
\hline \multirow{3}{*}{ Q-EG } & A & $42,301(6,310)^{* *}$ & 2,14 & 2,68 & $1,610 * *$ & $14,287 * *$ \\
\hline & $\mathrm{V}$ & $8,513(5,652) \mathrm{ns}$ & 11,24 & 13,22 & $1,649 * *$ & $10,510^{* *}$ \\
\hline & $\mathrm{D}$ & $0,095(0,027)^{* *}$ & $-2,82$ & $-3,72$ & $-0,639 * *$ & $2,872 * *$ \\
\hline \multirow{3}{*}{ E-EG } & A & $36,413(3,084)^{* *}$ & 0,98 & 1,13 & $0,378 * *$ & $0,312 * *$ \\
\hline & $\mathrm{V}$ & $8,321(2,910)^{* *}$ & 2,73 & 4,13 & $0,569 * *$ & $0,541 * *$ \\
\hline & $\mathrm{D}$ & $0,115(0,018)^{* * *}$ & $-1,51$ & $-1,78$ & $-0,201 * *$ & $0,075 \mathrm{~ns}$ \\
\hline
\end{tabular}

Estimativa (erro padrão), * e ** indicam significativo, a $5 \%$ e $1 \%$, pelo teste $\mathrm{t}$ e ns indica não significativo.

Todos os parâmetros foram significativamente diferentes de zero para o modelo Exponencial, em qualquer tratamento (Tabela 2 e 3). No entanto, para o modelo Quociente, a estimativa do tempo de meia vida ( $V$, dias) do K prontamente liberável $(A, \mathrm{mg})$ não diferiu de zero para os tratamentos LVAEB, LVAEE eLVAEG, ou seja, em termos práticos, houve liberação instantânea do K prontamente liberável. Da mesma forma, a taxa de lenta liberação de $\mathrm{K}\left(D, \mathrm{mg} \mathrm{kg}^{-1}\right.$ dia $\left.^{-1}\right)$ não foi diferente de zero para o modelo Quociente ajustado ao LVdfEE, o que pode apontar, na prática, que todo K passível de liberação é proveniente da fonte orgânica e que o solo não dificulta sua liberação. Tais diferenças em conclusão estatística, relacionadas aos modelos são devido ao fato de o modelo Quociente estimar menores valores para $V$ e $D$ e ainda apresentar maior valor para o erro padrão dessas estimativas se comparado ao modelo Exponencial.

$\mathrm{O}$ vício nas estimativas dos parâmetros foi menor para o modelo Exponencial que para o modelo Quociente, considerando todos os tratamentos (Tabela 2 e 3). Em geral, observou-se que o parâmetro $V$ apresenta maior vício que $A$ e $D$. Tal fato é devido a $V$ aparecer de forma não linear no modelo (SCHABENBERGER \& PIERCE, 2002).

$\mathrm{O}$ vício bootstrap apresentou valores próximos ao vício de Box (Tabela 2 e 3). Em geral, o vício bootstrap apresentou valores superiores, sendo que, para LVdfEC e LVdfEE, foram inferiores ao vício de Box. Tal resultado indica que o vício de Box estima bem o vício nas estimativas dos parâmetros e que, 
Tabela 3- Estimativas, erro padrão, vício de Box, vício bootstrap, assimetria e curtose para os parâmetros em função dos tratamentos para o solo LVdf.

\begin{tabular}{|c|c|c|c|c|c|c|}
\hline Mod.-Trat. & Parm. & Est(Err.Pad.) & V.Box $(\%)$ & V.Boot $(\%)$ & Assim. & Curto. \\
\hline \multirow{3}{*}{ Q-EB } & $\mathrm{A}$ & $51,500(3,235)^{* *}$ & 0,38 & 0,56 & $0,245^{* *}$ & $0,032 \mathrm{~ns}$ \\
\hline & $\mathrm{V}$ & $9,268(2,448)^{* *}$ & 1,87 & 2,25 & $0,466^{* *}$ & $0,350 * *$ \\
\hline & $\mathrm{D}$ & $0,089(0,014)^{* *}$ & $-0,65$ & $-1,24$ & $-0,122 \mathrm{~ns}$ & $-0,101 \mathrm{~ns}$ \\
\hline \multirow{3}{*}{ E-EB } & A & $43,443(1,720)^{* *}$ & 0,22 & 0,34 & $0,100 \mathrm{~ns}$ & $0,020 \mathrm{~ns}$ \\
\hline & $\mathrm{V}$ & $8,350(1,361)^{* *}$ & 0,6 & 0,95 & $0,340 * *$ & $0,239^{*}$ \\
\hline & $\mathrm{D}$ & $0,116(0,010)^{* *}$ & $-0,39$ & $-0,56$ & $-0,139 *$ & $0,046 \mathrm{~ns}$ \\
\hline \multirow{3}{*}{ Q-EC } & A & $117,982(17,645)^{* *}$ & 2,17 & 1,65 & $0,843^{* *}$ & $1,256^{* *}$ \\
\hline & $\mathrm{V}$ & $23,373(8,354)^{* *}$ & 5,14 & 3,6 & $0,882^{* *}$ & $1,307 * *$ \\
\hline & $\mathrm{D}$ & $0,127(0,061)^{*}$ & $-4,9$ & $-3,49$ & $-0,502 * *$ & $0,480 * *$ \\
\hline \multirow{3}{*}{ E-EC } & A & $87,527(7,302)^{* *}$ & 0,98 & 0,86 & $0,537 * *$ & $0,546^{* *}$ \\
\hline & $\mathrm{V}$ & $15,621(3,296)^{* *}$ & 1,79 & 1,45 & $0,589 * *$ & $0,791 * *$ \\
\hline & $\mathrm{D}$ & $0,212(0,039)^{* *}$ & $-1,78$ & $-1,62$ & $-0,354 * *$ & $0,280 * *$ \\
\hline \multirow{3}{*}{ Q-EE } & A & $163,966(20,670)^{* *}$ & 1,45 & 1,34 & $0,747 * *$ & $1,157 * *$ \\
\hline & $\mathrm{V}$ & $42,014(9,202)^{* *}$ & 2,25 & 1,88 & $0,711^{* *}$ & $0,975^{* *}$ \\
\hline & $\mathrm{D}$ & $0,062(0,060) \mathrm{ns}$ & $-7,78$ & $-7,97$ & $-0,489 * *$ & $0,558 * *$ \\
\hline \multirow{3}{*}{ E-EE } & A & $109,571(8,171)^{* *}$ & 0,72 & 0,63 & $0,450 * *$ & $0,354 * *$ \\
\hline & $\mathrm{V}$ & $23,961(3,228)^{* *}$ & 0,91 & 0,79 & $0,426 * *$ & $0,392^{* *}$ \\
\hline & $\mathrm{D}$ & $0,188(0,039) * *$ & $-1,67$ & $-1,36$ & $-0,362 * *$ & $0,201^{*}$ \\
\hline \multirow{3}{*}{ Q-EG } & A & $76,714(7,829)^{* *}$ & 1,02 & 1,17 & $0,510^{* *}$ & $0,740 * *$ \\
\hline & $\mathrm{V}$ & $17,264(5,017) * *$ & 3,06 & 3,6 & $0,464 * *$ & $0,513 * *$ \\
\hline & $\mathrm{D}$ & $0,130(0,029)^{* *}$ & $-1,59$ & $-1,97$ & $-0,250 * *$ & $0,247^{*}$ \\
\hline \multirow{3}{*}{ E-EG } & A & $59,984(3,406)^{* *}$ & 0,46 & 0,54 & $0,290^{* *}$ & $0,358 * *$ \\
\hline & $\mathrm{V}$ & $12,834(2,145)^{* *}$ & 1,00 & 1,3 & $0,191 * *$ & $0,141 \mathrm{~ns}$ \\
\hline & $\mathrm{D}$ & $0,180(0,019)^{* *}$ & $-0,7$ & $-0,93$ & $-0,082 \mathrm{~ns}$ & $0,199 *$ \\
\hline
\end{tabular}

Estimativa (erro padrão), * e ** indicam significativo, a $5 \%$ e $1 \%$, pelo teste t e ns indica não significativo.

portanto, poderia ser usado, dado o maior custo computacional para obtenção do vício bootstrap.

O modelo Exponencial apresentou menores desvios de assimetria e curtose para as amostras bootstrap dos parâmetros que o modelo Quociente, o que indica comportamento mais próximo do linear (Tabela 2 e 3). Dentre os parâmetros, $D$ foi o que apresentou, de forma geral, menores valores para assimetria e curtose. Segundo GILLS \& RATKOWSKY (1978), tal resultado é devido a $D$ aparecer linearmente no modelo. Os desvios de assimetria indicaram distribuição assimétrica à direita para $A \mathrm{e} V$, e assimetria à esquerda para $D$. Tal resultado indica que reparametrização com o uso de funções monótonas decrescentes poderiam corrigir os desvios para $A$ e $V$, sendo o contrário para $D$.

\section{CONCLUSÃO}

O modelo Exponencial apresentou menor não linearidade que o modelo Quociente por todas as medidas empregadas: curvatura média, vício de Box e estudo de simulação bootstrap. Portanto, o modelo Exponencial, do ponto de vista inferencial, foi mais adequado.

\section{REFERÊNCIAS}

BARROZO, M.A.S. et al. A study of the statistical discrimination of the drying kinetics equations. Food and Bioproducts Processing, Dorchester, v.82, p.219-225, 2004. Disponível em: <http://www.sciencedirect.com/science/article/pii/ S0960308504704368>. Doi: http://dx.doi.org/10.1205/ fbio.82.3.219.44176. Acesso em: $10 \mathrm{dez} .2009$.

BATES, D.M.; WATTS, D.G. Relative curvature measures of nonlinearity. Journal of the Royal Statistical Society. Serie B. Methodological, London, v.42, n.1, p.1-25, 1980. Disponível em: <http://www.jstor.org/stable/2984733>. Acesso em: 10 dez. 2009.

BOX, M.J. Bias in nonlinear estimation. Journal of Royal Statistical Society. Serie B. Methodological, London, v.33, n.2, p.171-201, 1971. Disponível em: <http://www.jstor.org/ stable/2985002>. Acesso em: 10 dez. 2009.

CABRERA, M.L. Modelling the flush of nitrogen mineralization caused by drying and rewetting soils. Soil Science Society of American Journal, Madison, v.57, n.1, p.63-66, 1993. Disponível em: <https://www.soils.org/publications/sssaj/ abstracts/57/1/SS0570010063>. Acesso em: 10 dez. 2009. 
CANESIN, R.C.F.S; CORREA, L. de S. Uso de esterco associado à adubação mineral na produção de mudas de mamoeiro (Carica papaya L.). Revista Brasileira de Fruticultura, Jaboticabal, v.28, n.3, p.481-486, 2006. Disponível em: <http://dx.doi.org/ 10.1590/S0100-29452006000300031>. Acesso em: 10 dez. 2009.

CAMARGO, F.A. de O. et al. Empirical models to predict soil nitrogen mineralization. Ciência Rural, Santa Maria, v.32, n.3, p.393-399, 2002. Disponível em: <http://dx.doi.org/10.1590/ S0103-84782002000300005>. Acesso em: 10 dez. 2009.

GILLS, P.R.; RATKOWSKY, D.A. The behavior of estimators of the parameters of various yield-density relationships. Biometrics, Washington, v.34, n.2, p.191-198, 1978. Disponível em: <http://www.jstor.org/stable/2530009>. Acesso em: 10 dez. 2009.

MALAVOLTA, E. Avaliação do estado nutricional. In: MALAVOLTA, E. Elementos de nutrição mineral de plantas. São Paulo: Ceres, 1980. p.219-251.

MAZUCHELI, J.; ACHCAR, J.A. Algumas considerações em regressão não linear. Acta Scientiarum, Maringá, v.24, n.6, p.1761-1770, 2002. Disponível em: <http://eduem.uem.br/ ojs/index.php/ActaSciTechnol/article/view/251>. Acesso em: 10 dez. 2009.

MENDES, P.N. et al. Modelo logístico difásico no estudo do crescimento de fêmeas da raça Hereford. Ciência Rural, Santa Maria, v.38, n.7, p.1984-1990, 2008. Disponível em: <htt://dx.doi.org/10.1590/S0103-84782008000700029>. Acesso em: 10 dez. 2009.
NACHTIGALL, G.R.; RAIJ, B. Análise e interpretação de potássio no solo. In: YAMADA, T.; ROBERTS, T.L. Potássio na agricultura brasileira. Piracicaba: Potafós, 2005. p.21-32.

OLIVEIRA, W.S.J. Liberação de potássio em latossolos tratados com resíduos orgânicos. 2008. 45f. Dissertação (Mestrado em Ciência do Solo) - Curso de Pós-graduação em Ciência do Solo, Universidade Federal de Lavras, MG.

PEREIRA, J.M. et al. Nonlinear models to predict nitrogen mineralization in an Oxisol. Scientia Agricola, Piracicaba, v.62, n.4, p.395-400, 2005. Disponível em: <http://dx.doi.org/10.1590/ S0103-90162005000400014>. Acesso em: 10 dez. 2009.

RATKOWSKY, D.A. Nonlinear regression modeling. New York: M. Dekker, 1983. 276p.

R Development Core Team. R: a language and environment for statistical computing. Vienna: R Foundation for Statistical Computing, 2009. ISBN 3-900051-07-0, URL. Disponível em: <http://www.R-project.org/>. Acesso em: 10 dez. 2009.

SCHABENBERGER, O.; PIERCE, F.J. Comtemporary statistical models for the plant and soil science. Boca Raton: CRC, 2002. 738p.

SEBER, G.A.F.; WILD, C.J. Nonlinear regression. New York: J. Wiley, 1989. 752p.

ZEVIANI, W.M. Avaliação de modelos de regressão não linear na cinética de liberação de potássio de resíduos orgânicos. 2009. 85f. Dissertação (Mestrado em Estatística e Experimentação Agropecuária) - Curso de Pós-graduação em Estatística e Experimentação Agropecuária, Universidade Federal de Lavras, MG. 